\title{
NUMERICAL STUDIES ON STABILITY OF SLENDER SUPPORTING STRUCTURES
}

\author{
Krzysztof Sokót, Sebastian Uzny \\ Institute of Mechanics and Machine Design Foundation, Czestochowa University of Technology \\ Częstochowa, Poland \\ sokol@imipkm.pcz.pl,uzny@imipkm.pcz.pl
}

\begin{abstract}
In this paper the results of studies on stability of the geometrically non-linear column (slender system) composed of two rods have been presented. The supporting structure has a defect in the form of cracks which are present in each of rods. The cracks are simulated by means of rotational springs. On the basis of the total potential energy principle, the boundary problem is being formulated. The results show an influence of the crack size on the stability of the column in particular on bifurcation load magnitude.
\end{abstract}

Keywords: stability, cracks, bifurcation load, slender systems, column

\section{Introduction}

The considered columns are classified as slender supporting systems due to relation between cross-section dimensions and the total length of the structure. In these types of systems the most undesirable phenomena are: flutter instability, buckling and non-axially applied external load. The presence of the cracks is also very dangerous due to its influence on the dynamic as well as on static behaviour of the structure. At the static investigations the presence of a crack affects the loading capacity, but what is important is that the capacity may differ depending on crack location.

The crack influence on stability as well as static and dynamic behavior, was discussed and presented in many scientific papers in recent years. Dimarogonas and Anifantis in [1] have studied the stability of beam structures subjected to follower and vertical loads. The crack was modelled by a general flexibility matrix which expressed the local flexibility of the beam. The same author in [2] introduced the Rayleigh principle for an estimation of the change of the natural frequencies as well as modes of vibration of the structure with a crack. Bergman and Lee [3] have studied the stepped beams and rectangular plates. Chondros [4] has performed theoretical, numerical and experimental studies of cracked aluminum beams. Binici in [5] has studied single beams in which the multiple cracks were modeled with rotational springs. Sokół [6] presented investigations on an influence of single crack presence in the cantilever column on vibrations and stability as well as on the 
amplitude - vibration frequency relationship. Mostly the traditional analysis [7] was performed but Sokól and Kulawik [8] have proposed an implementation of genetic algorithms in order to find optimum parameters of the system at which the structure will be the least sensitive to the crack presence. In the literature two different types of problems are studied that take cracks into account. The first one is focused on linear problems where the cracks always remain open. The second one takes into account the non-linear problems where the crack opens and closes in time. In the numerical simulations of cracks the different approach can be found: reduction of the cross-section area, definition of complex mathematical functions or introduction of rotational springs. The studies performed by Chondros et al. [9] and Arif Gurel [10] show that despite rotational spring simplicity the comparison of the data of numerical simulations and experimental studies leads to accuracy of good results.

In most scientific papers, the single linear columns and beams with cracks are presented. In this paper, the multi-member non-linear column with a crack in each of rods is investigated. The boundary problem of multi-member systems is formulated on the basis of the non-linear differential equations. In this case, the non-linear relation strain - transversal deflection is used. The systems are characterized by the presence of rectilinear and curvilinear form of static equilibrium. The presence of both forms depends on the magnitude of the applied external load. Many scientific papers can be found where the phenomenon of the change of the static equilibrium form takes place along with the presence of the local and global instability regions (see $[11,12])$. The external load is being realized by means of an axially applied external force with a constant line of action (Euler's load). The proposed method of boundary problem formulation can be easily adapted to the more complicated structures, such as flat frames composed of n-elements. The main purpose of this paper is to present an influence of the crack size and location on loading capacity. The considered crack is present in both rods of the structure. Due to investigations of a multi-cracked system the contour graphs are used in order to achieve the best method of presentation. The results of simulations allow one to see the areas of special care in which the appearance of the crack will quickly lead to destruction of the structure.

\section{Boundary problem formulation}

The investigated structure is presented in Figure 1. Two cracks simulated by means of rotational springs $C_{w}$ and $C_{z}$ with linear characteristics are present. It is assumed that the cracks remain open and they divide the rods into four elements indexed as $1,2,3,4$. The total length of the column is $l=l_{1}+l_{3}=l_{2}+l_{4}$. The external compressive axially applied force (Euler's load) is placed on the free end of the system. The considered system can be composed of two coaxial tubes, tube and rod or be a flat frame with the infinite connection stiffness of the end points of rods (3) and (4). 


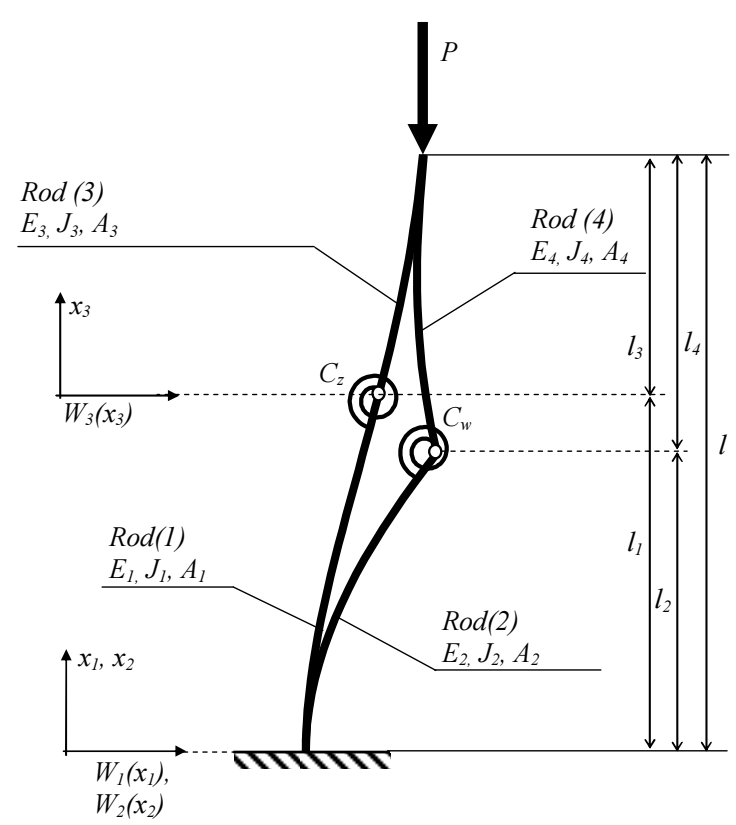

Fig. 1. Investigated system

The boundary problem has been formulated on the basis of static criterion of stability $(\delta V=0)$ where the potential energy $V$ of the system is given by the following formula:

$$
\begin{aligned}
& V=\frac{1}{2} \sum_{i=1}^{4}(E J)_{i} \int_{0}^{l_{i}}\left[\frac{d^{2} W_{i}(x)}{d x_{i}^{2}}\right]^{2} d x+\frac{1}{2} \sum_{i=1}^{4}(E A)_{i} \int_{0}^{l_{i}}\left[\frac{d U_{i}\left(x_{i}\right)}{d x_{i}}+\frac{1}{2}\left(\frac{d W_{i}\left(x_{i}\right)}{d x_{i}}\right)^{2}\right]^{2} d x+ \\
& +\frac{1}{2} C_{z}\left(\left.\frac{d W_{1}\left(x_{1}\right)}{d x_{1}}\right|^{x_{1}=l_{1}}-\left.\frac{d W_{3}\left(x_{3}\right)}{d x_{3}}\right|_{x_{3}=0}\right)^{2}+\frac{1}{2} C_{w}\left(\left.\frac{d W_{2}\left(x_{2}\right)}{d x_{2}}\right|^{x_{2}=l_{2}}-\left.\frac{d W_{4}\left(x_{4}\right)}{d x_{4}}\right|_{x_{4}=0}\right)^{2}+ \\
& +P U_{3}(l)
\end{aligned}
$$

The geometrical boundary conditions of the column are:

$$
\begin{gathered}
\left.W_{1}\left(x_{1}\right)\right|_{x_{1}=0}=\left.W_{2}\left(x_{2}\right)\right|_{x_{2}=0}=0 \\
\left.\frac{\partial W_{1}\left(x_{1}\right)}{\partial x_{1}}\right|_{x_{1}=0}=\left.\frac{\partial W_{2}\left(x_{2}\right)}{\partial x_{2}}\right|_{x_{2}=0}=\left.0 \quad \frac{\partial W_{3}\left(x_{3}\right)}{\partial x_{3}}\right|^{x_{3}=l_{3}}=\left.\frac{\partial W_{4}\left(x_{4}\right)}{\partial x_{4}}\right|^{x_{4}=l_{4}} \\
\left.W_{1}\left(x_{1}\right)\right|^{x_{1}=l_{1}}=\left.\left.W_{3}\left(x_{3}\right)\right|_{x_{3}=0} W_{2}\left(x_{2}\right)\right|^{x_{2}=l_{2}}=\left.\left.W_{4}(0)\right|_{x_{4}=0} W_{3}\left(x_{3}\right)\right|^{x_{3}=l_{3}}=\left.W_{4}\left(x_{4}\right)\right|^{x_{4}=l_{4}} \text { (2a-h) }
\end{gathered}
$$


On the basis of variation and integration operations performed on (1) into which the geometrical boundary conditions are introduced $(2 \mathrm{a}, \mathrm{g})$, the natural boundary conditions can be found:

$$
\begin{aligned}
& \left.(E J)_{3} \frac{\partial^{2} W_{3}\left(x_{3}\right)}{\partial x_{3}^{2}}\right|^{x_{3}=l_{3}}+\left.(E J)_{4} \frac{\partial^{2} W_{4}\left(x_{4}\right)}{\partial x_{4}^{2}}\right|^{x_{4}=l_{4}}=0 \\
& \left.(E J)_{3} \frac{\partial^{3} W_{3}\left(x_{3}\right)}{\partial x_{3}^{3}}\right|^{x_{3}=l_{3}}+\left.P \frac{\partial W_{3}\left(x_{3}\right)}{\partial x_{3}}\right|^{x_{3}=l_{3}}+\left.(E J)_{4} \frac{\partial^{3} W_{4}\left(x_{4}\right)}{\partial x_{4}^{3}}\right|^{x_{4}=l_{4}}=0 \\
& \left.(E J)_{1} \frac{\partial^{3} W_{1}\left(x_{1}\right)}{\partial x_{1}^{3}}\right|^{x_{1}=l_{1}}+\left.S_{1} \frac{\partial W_{1}\left(x_{1}\right)}{\partial x_{1}}\right|^{x_{1}=l_{1}}-\left.(E J)_{3} \frac{\partial^{3} W_{3}\left(x_{3}\right)}{\partial x_{3}^{3}}\right|_{x_{3}=0}-\left.S_{3} \frac{\partial W_{3}\left(x_{3}\right)}{\partial x_{3}}\right|_{x_{3}=0}=0 \quad(3 \mathrm{c}) \\
& \left.(E J)_{2} \frac{\partial^{3} W_{2}\left(x_{2}\right)}{\partial x_{2}^{3}}\right|^{x_{2}=l_{2}}+\left.S_{2} \frac{\partial W_{2}\left(x_{2}\right)}{\partial x_{2}}\right|^{x_{2}=l_{2}}-\left.(E J)_{4} \frac{\partial^{3} W_{4}\left(x_{4}\right)}{\partial x_{4}^{3}}\right|_{x_{4}=0}-\left.S_{4} \frac{\partial W_{4}\left(x_{4}\right)}{\partial x_{4}}\right|_{x_{4}=0}=0 \\
& -\left.(E J)_{3} \frac{\partial^{2} W_{3}\left(x_{3}\right)}{\partial x_{3}^{2}}\right|_{x_{3}=0}+C_{z}\left[\left.\frac{\partial W_{3}\left(x_{3}\right)}{\partial x_{3}}\right|_{x_{3}=0}-\left.\frac{\partial W_{1}\left(x_{1}\right)}{\partial x_{1}}\right|^{x_{1}=l_{1}}\right]=0 \\
& \left.(E J)_{1} \frac{\partial^{2} W_{1}\left(x_{1}\right)}{\partial x_{1}^{2}}\right|^{x_{1}=l_{1}}-C_{z}\left[\left.\frac{\partial W_{3}\left(x_{3}\right)}{\partial x_{3}}\right|_{x_{3}=0}-\left.\frac{\partial W_{1}\left(x_{1}\right)}{\partial x_{1}}\right|^{x_{1}=l_{1}}\right]=0 \\
& -\left.(E J)_{4} \frac{\partial^{2} W_{4}\left(x_{4}\right)}{\partial x_{4}^{2}}\right|_{x_{4}=0}+C_{w}\left[\left.\frac{\partial W_{4}\left(x_{4}\right)}{\partial x_{4}}\right|_{x_{4}=0}-\left.\frac{\partial W_{2}\left(x_{2}\right)}{\partial x_{2}}\right|^{x_{2}=l_{2}}\right]=0 \\
& \left.(E J)_{2} \frac{\partial^{2} W_{2}\left(x_{2}\right)}{\partial x_{2}^{2}}\right|^{x_{2}=l_{2}}-C_{w}\left[\left.\frac{\partial W_{4}\left(x_{4}\right)}{\partial x_{4}}\right|_{x_{4}=0}-\left.\frac{\partial W_{2}\left(x_{2}\right)}{\partial x_{2}}\right|^{x_{2}=l_{2}}\right]=0 \\
& S_{2}=S_{4} \quad S_{1}+S_{2}=\left.P \quad U_{1}\left(x_{1}\right)\right|_{x_{1}=0}=\left.U_{2}\left(x_{2}\right)\right|_{x_{2}=0}=0 \\
& \left.U_{1}\left(x_{1}\right)\right|^{x_{1}=l_{1}}=\left.\left.U_{3}(0)\right|_{x_{3}=0} \quad U_{2}\left(x_{2}\right)\right|^{x_{2}=l_{2}}=\left.\left.U_{4}(0)\right|_{x_{4}=0} \quad U_{3}\left(x_{3}\right)\right|^{x_{3}=l_{3}}=\left.U_{4}\left(x_{4}\right)\right|^{x_{4}=l_{4}} \quad \text { (3m-o) }
\end{aligned}
$$

Besides natural boundary conditions the differential equation of transversal displacements (4) and longitudinal ones (5) are obtained:

$$
(E J)_{i} \frac{d^{4} W_{i}\left(x_{i}\right)}{d x_{i}^{4}}+S_{i} \frac{d^{2} W_{i}\left(x_{i}\right)}{d x_{i}^{2}}=0 \quad i=1,2,3,4
$$




$$
U_{i}\left(x_{i}\right)-U_{i}(0)=-\frac{S_{i}}{(E A)_{i}} x_{i}-\int_{0}^{x_{i}}\left(\frac{d W_{i}\left(x_{i}\right)}{d x_{i}}\right)^{2} d x_{i} \quad i=1,2,3,4
$$

where $S_{i}$ is an internal longitudinal force. The solution of (4) has been adopted in the form:

$$
W_{i}\left(x_{i}\right)=A_{i} \cos \left(k x_{i}\right)+B_{i} \sin \left(k x_{i}\right)+C_{i} x_{i}+D_{i}
$$

The bifurcation load magnitude as well as an influence of the crack size on the stability of the column is obtained after an introduction of (6) into boundary conditions. This operation leads to a matrix determinant, which equated to zero, creates a transcendental equation used in numerical simulations.

\section{Results of numerical simulations}

The results of numerical simulations are presented in the non-dimensional form where the following markings are introduced: magnitude of bifurcation load (7a), crack size (7b, c), crack location (7d, e), coefficient of flexural rigidity (7f):

$$
\begin{aligned}
p & =\frac{P l^{2}}{(E J)_{1}+(E J)_{2}}, c w=\frac{C_{w} l}{(E J)_{2}+(E J)_{4}}, c z=\frac{C_{z} l}{(E J)_{1}+(E J)_{3}}, d_{w}=\frac{l_{2}}{l}, d_{z}=\frac{l_{1}}{l}, \\
\mu & =\frac{(E J)_{2}}{(E J)_{1}}
\end{aligned}
$$

In Figures 2-5, two different ranges of the presentation results were used.
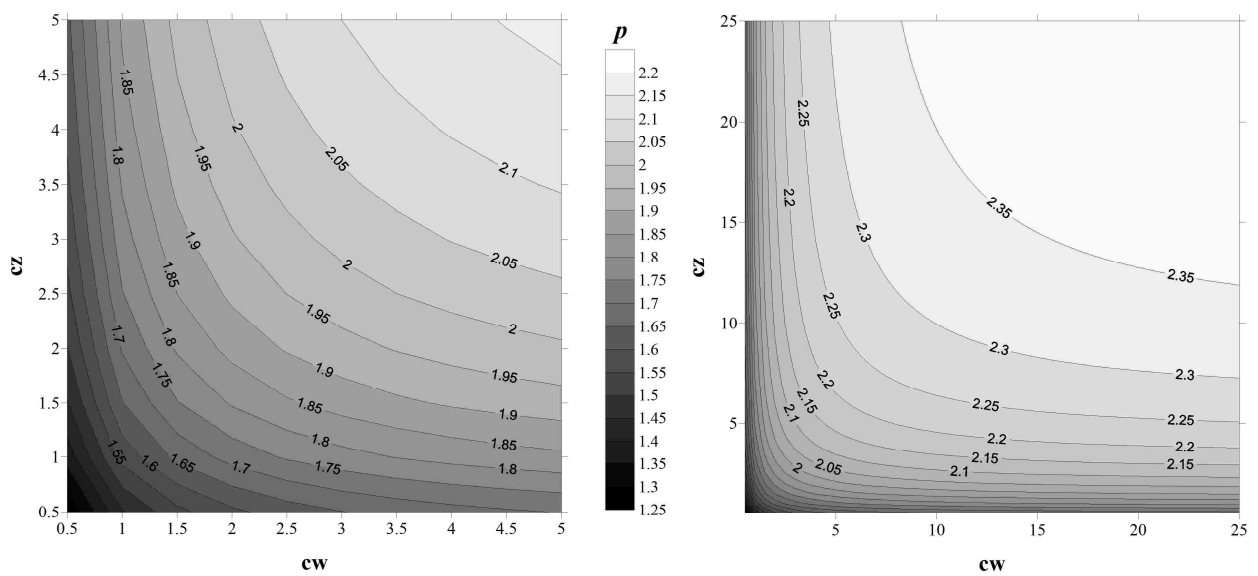

Fig. 2. Influence of cracks on bifurcation load magnitude; data: $\mu=1, d_{w}=0.5, d_{z}=0.01$ 

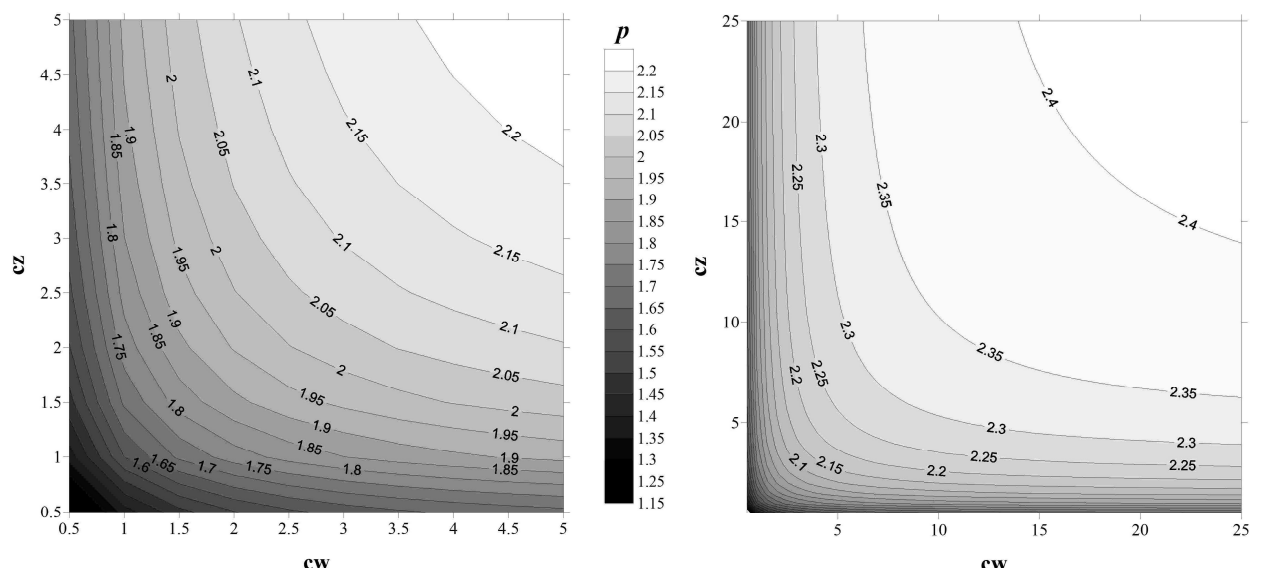

Fig. 3. Influence of the cracks on bifurcation load magnitude; data: $\mu=1, d_{w}=d_{z}=0.5$
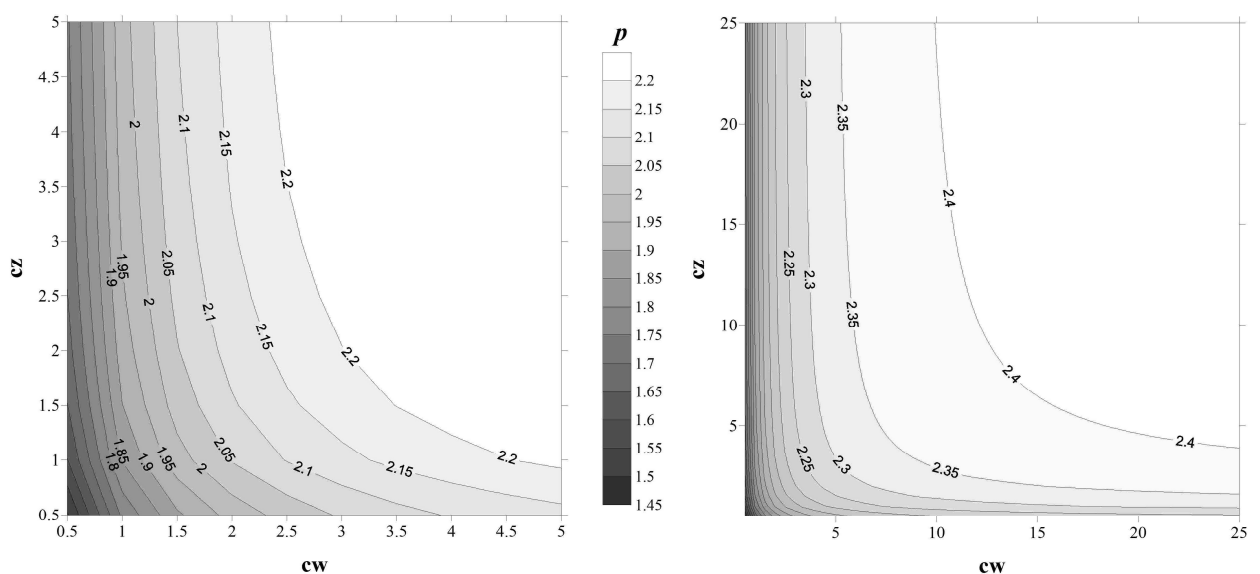

Fig. 4. Influence of the cracks on bifurcation load magnitude; data: $\mu=1, d_{w}=0.5, d_{z}=0.75$
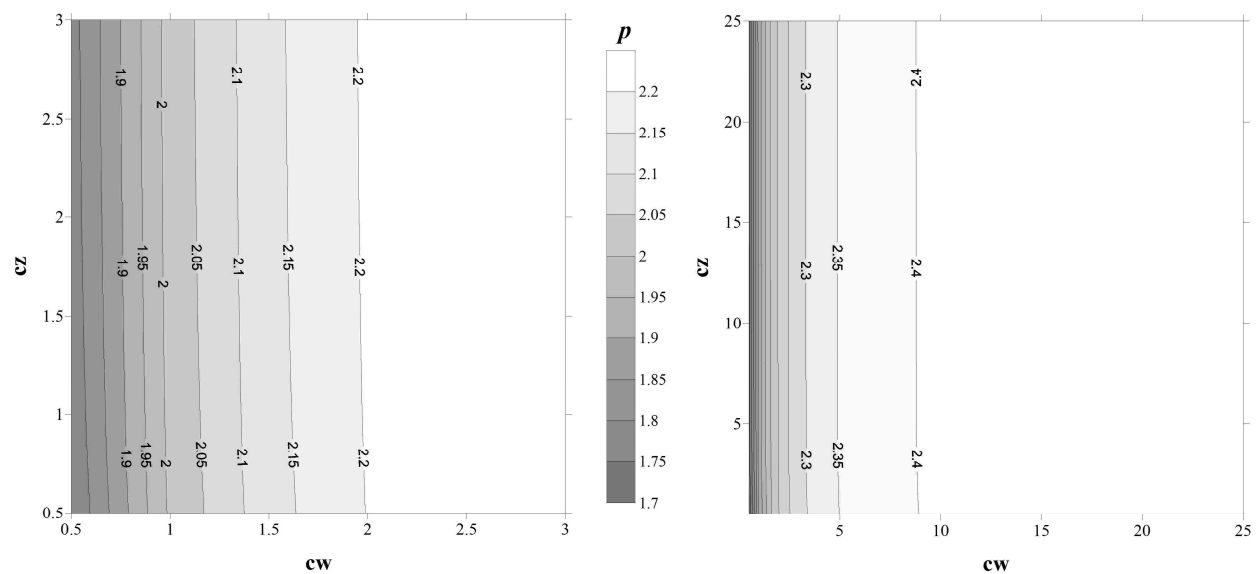

Fig. 5. Influence of the cracks on bifurcation load magnitude; data: $\mu=1, d_{w}=0.5, d_{z}=0.99$ 
The parameters $p, c w, c z$ are related to the total stiffness of the system. This type of substitution is widely used in the presentation of the results and gives a great comparison opportunity of the systems loaded by the same type of an external force but at different parameters of the structure in which the crack is present. In the simulations it is assumed that: $(E J)_{1}=(E J)_{3},(E J)_{2}=(E J)_{4}$.

Because of a large number of results and different configurations, for the general presentation and description the following locations of cracks have been chosen: $d_{w}=0.5$ and $d_{z}=0.01,0.5,0,75,0.99$. Additionally considered crack sizes are $0.1<c w<100,0.1<c z<100$ and $\mu=0.2,0.5,1$.

Taking into account the results from Figure 2, it can be assumed that the lowest magnitude of bifurcation load at this configuration $(p=1.25)$ appears at the smallest stiffness of rotational springs (highest crack size). It must be stated that the lowest loading capacity is not the lowest one that can be observed (see Fig. 3). The contours are unsymmetrical due to different locations of the cracks. The greater the rotational spring stiffness, the higher the loading capacity of the column. The bifurcation load area, unaffected by cracks can be found at $p=\pi^{2} / 4$ (at $c w>100, c z>100$ ) non-dimensional loading capacity of a cantilever column subjected to Euler's load. In Figure 3, the cracks are placed symmetrically at $d_{w}=d_{z}=0.5$, which results in symmetrical data distribution but, what is most important, the loading capacity decreases with respect to Figure 2, down to $p=1.16$ (the lowest loading capacity). With the appearance of the crack at $d_{z}=0.75$ (Fig. 4) an increase of the lowest loading capacity is present $(p=1.49)$.

An analysis of Figure 5 shows that the crack presence near the free end of the column $\left(d_{z}=0.99\right)$ has small influence on bifurcation load magnitude, regardless of its size. The lowest bifurcation load magnitude is $p=1.75$. It can be assumed that an increase of the unaffected area results in more a rapid loading capacity decrease and that the minimum loading capacity decreases at the beginning (crack "shifts" from the fixation in the direction of the free end) and after reaching the critical point an increase can be observed. The location of the critical point depends on crack location in the other rod as well as on coefficient of flexural rigidity.

The change of bifurcation load magnitude in relation to the coefficient of flexural rigidity is presented in Figures 6 and 7 at $\mu=0.5$ and $\mu=0.2$, respectively. According to the results presented in Figure 3 the loading capacity drop is symmetrical but when the reduction of coefficient of flexural rigidity is investigated the drop becomes unsymmetrical and more rapid. The lowest loading capacity is also smaller than it is in the symmetrical case (see Fig. 3). 

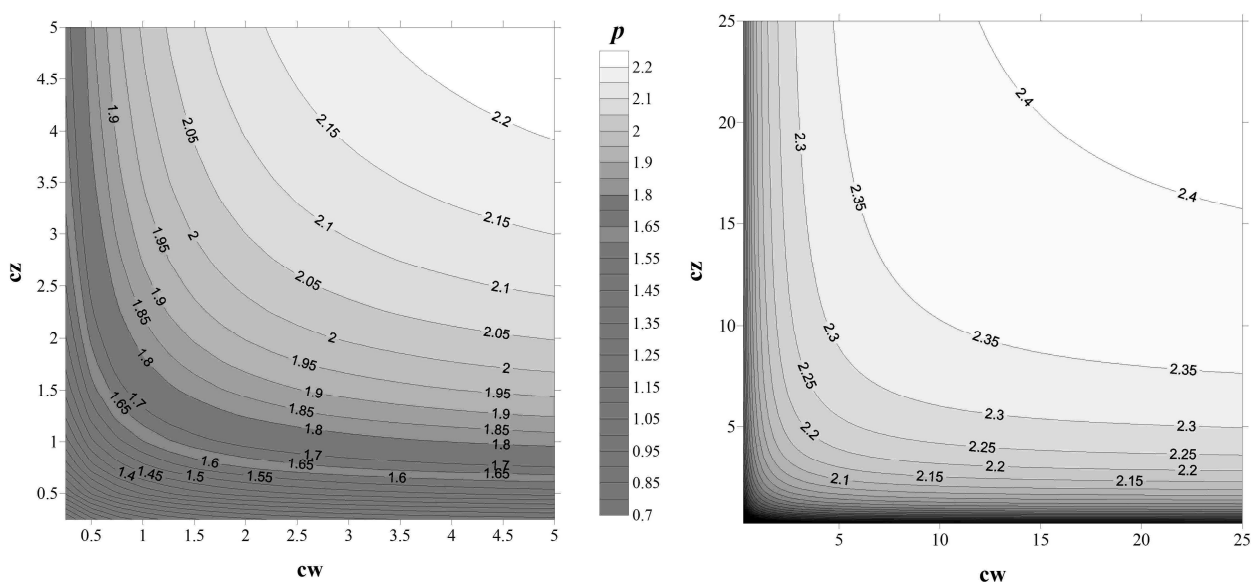

Fig. 6. Influence of the cracks on bifurcation load magnitude at a different bending rigidity factor; other data: $d_{w}=0.5, d_{z}=0.5, \mu=0.5$
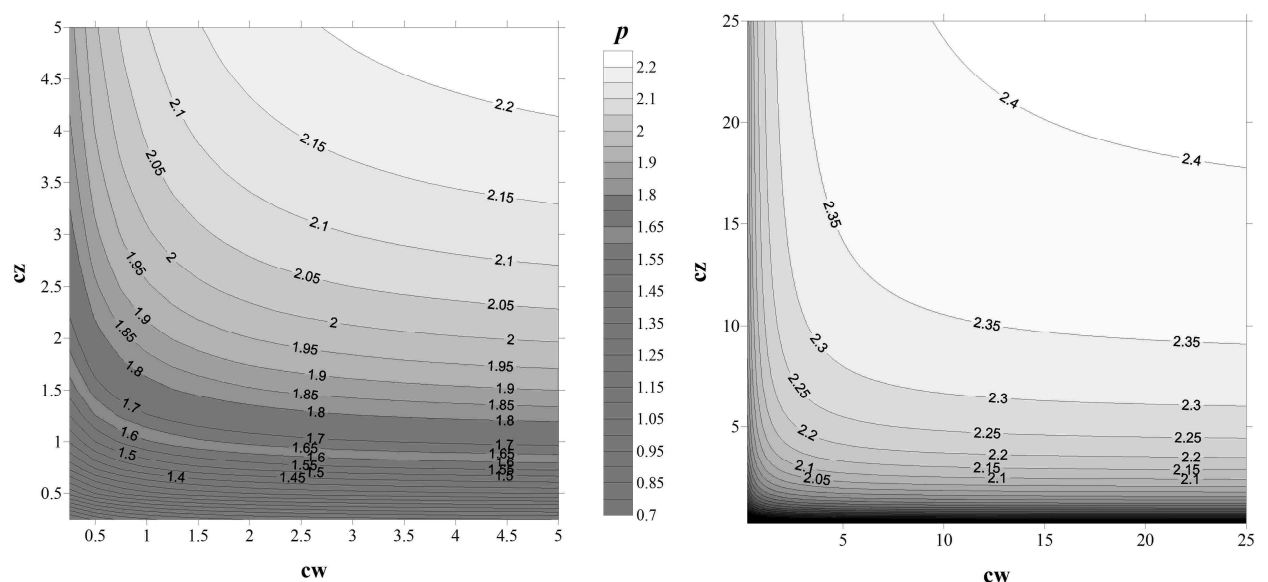

Fig. 7. Influence of the cracks on bifurcation load magnitude at a different bending rigidity factor; other data: $d_{w}=0.5, d_{z}=0.5, \mu=0.2$

\section{Conclusions}

In this paper the geometrically non-linear column used as a slender supporting structure has been investigated. The system was loaded by Euler's force. The structure is composed of two rods in which the cracks are present. In the simulations of cracks, the rotational springs have been used. The detailed description of the results can be found in Section 3. On the basis of the analysis of the results of numerical simulations, the general conclusions are as follows:

- depending on the crack location, its presence reduces the loading capacity of the system in different level, 
- the size of unaffected by cracks area (part of the $c w-c z$ graph for which the drop of loading capacity is smaller than 1\%) highly depends on the crack location,

- the value of bifurcation load changes nonlinearly with respect to the location of the crack,

- the change of loading capacity highly depends on coefficient of flexural rigidity.

When the non-linear systems are investigated, it can be stated that at higher flexural rigidity asymmetry the defect on the stiffer rod will lead to greater reduction of bifurcation load magnitude than the defect of the less rigid one while with small asymmetry, damage to any rod will have a similar influence on load bifurcation. Due to a very complicated problem and a large number of results, the presented plots are only a small sample of data used in structure monitoring. In the future, the investigations on dynamic characteristics should be discussed and experimental studies should be done in order to verify the correctness of the proposed mathematical models.

\section{References}

[1] Anifantis N., Dimarogonas A.D., Stability of columns with a single crack subjected to follower and axial loads, International Journal of Solids and Structures 1999, 19, 281-291.

[2] Chondros T.G., Dimarogonas A.D., Dynamic sensitivity of structures to cracks, Journal of Vibration, Acoustics, Stress and Reliability in Design 1981, 111, 251-256.

[3] Lee J., Bergman L.A., The vibration of stepped beams and rectangular plates by an elemental dynamic flexibility method, Journal of Sound and Vibration 1994, 171, 617-640.

[4] Chondros T.G., The continuous crack flexibility model for crack identification, Fatigue \& Fracture of Engineering Materials \& Structures 2001, 24, 643-650.

[5] Binici B., Vibration of beams with multiple open cracks subjected to axial force, Journal of Sound and Vibration 2005, 287, 277-295.

[6] Sokół K., Linear and nonlinear vibrations of a column with an internal crack, Journal of Engineering Mechanics 2014, 140(5), http://dx.doi.org/10.1061/(ASCE)EM.1943-7889.0000719

[7] Sokół K., Uzny S., Instability and vibration of multi-member columns subjected to Euler's load, Archive of Applied Mechanics 2015, DOI: 10.1007/s00419-015-1068-6.

[8] Sokół K., Kulawik A., Optimization of slender systems by means of genetic algorithms, Journal of Applied Mathematics and Computational Mechanics 2014, 13(1), 115-125.

[9] Chondros T.G., Dimarogonas A.D., Yao J., A continuous cracked beam vibration theory, Journal of Sound and Vibration 1998, 215, 17-34.

[10] Arif Gurel M., Buckling of slender prismatic circular columns weakened by multiple edge cracks, Acta Mechanica 2007, 188, 1-19.

[11] Tomski L., Uzny S., Vibration and stability of geometrically non-linear column subjected to generalised load by a force directed towards the positive pole, International Journal of Structural Stability and Dynamics 2008, 8(1), 1-24.

[12] Tomski L., Uzny S., Free vibration and the stability of a geometrically non-linear column loaded by a follower force directed towards the positive pole, International Journal of Solids and Structures 2008, 45(1), 87-112. 